\section{MS13-O4 Zinc dependent \\ 3'-nucleases/nucleotidases from plants, fungi and bacteria - varied roles and specificity}

Jan Dohnalek ${ }^{1}$, Tomas Koval ${ }^{1}$, Lars H. Østergaard ${ }^{2}$, Petra Lipovova $^{3}$, Jarmila Duskova ${ }^{1}$, Tereza Skalova ${ }^{1}$, Maria Trundova ${ }^{1}$, Karla Fejfarova ${ }^{1}$, Jan Stransky $^{1}$

1. Institute of Biotechnology CAS, Biocev, Průmyslová 595, 252 50 Vestec, Czech Republic

2. Novozymes A/S, Krogshoejvej 36, 2880 Bagsvaerd, Denmark

3. Institute of Chemical Technology, Technická 5, 16628 Prague, Czech Republic

email: dohnalek@ibt.cas.cz

S1-P1 3'-nucleases/nucleotidases (EC 3.1.30.1) are small, mostly alpha-helical enzymes relying on the active centre formed by three zinc ions and surrounded by varied nucleotide binding sites [1]. These extracellular enzymes are employed in various roles in plants, fungi and bacteria but typically not in mammals. These enzymes are utilized in plants in apoptotic processes, tissue development and senescence, in protozoan parasites for securing nutrients and a similar role is expected in some gram-negative bacteria. The purpose of the enzyme in a given organism seems to lead to modification of substrate specificity. Tomato TBN1 has almost universal ability to cleave nucleic acids - single strand, double strand DNA, RNA and structured RNA and this is reflected by the appearance of the enzyme surface [2, 3]. Fungal S1 nuclease shows basically strict ssDNA preference. The enzyme specificity is defined by the width of the cleft, presence of nucleotide binding sites and distribution of electrostatic potential.

We have determined a series of complexes with a fungal enzyme showing a range of ligand-protein interactions. The required enzyme specificity/substrate promiscuity is connected with particular arrangement of surface sites. S1-P1 nuclease is also present in gram negative bacteria and protozoan parasites pathogenic to humans [e.g. 4]. We have cloned, expressed and purified nuclease from a human pathogen and proved its activity. Recent results leading aimed at clarification of specificity features, stabilization of the enzyme in bacteria and inhibition potential, will be presented.

The project is supported by MEYS CR (LG14009, LQ1604 NPU II), CZ.1.05/1.1.00/02.0109 BIOCEV provided by ERDF and MEYS, and CSF (15-05228S).

\section{References}

1. Romier C., Dominguez R., Lahm A., et al. Proteins 1998, vol. 32, no. 4, 414-424.

2. Koval' T., Lipovová P., Podzimek T., et al. Acta Cryst. 2013, vol. D69, no. 2, 213-226.

3. Stránský J., Koval' T., Podzimek T., et al. Acta Cryst. 2015, vol. F71, 1408-1415.

4. Guimarães-Costa A.B., DeSouza-Vieira T.S., Paletta-Silva R., et al. Infect. Immun. 2014, vol. 82, no. 4, 1732-40.

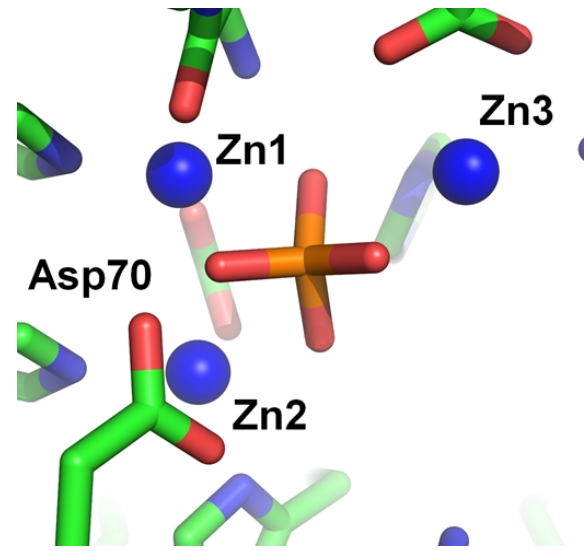

Figure 1. Complex of TBN1 and phosphate ion: mimic of product binding mode (PDB ID 4JDG).

Keywords: nuclease, 3'nuclease, zinc, S1-P1 nuclease, pathogen, substrate promiscuity 